Journal Club

Editor's Note: These short, critical reviews of recent papers in the Journal, written exclusively by graduate students or postdoctoral fellows, are intended to summarize the important findings of the paper and provide additional insight and commentary. For more information on the format and purpose of the Journal Club, please see http://www.jneurosci.org/misc/ifa_features.shtml.

\title{
The A $\beta$-Induced NFAT Apoptotic Pathway Is Also Activated by GSK-3 Inhibition: Implications for Alzheimer Therapeutics
}

\author{
Scott Ayton ${ }^{1 *}$ and Peng Lei ${ }^{1,2 *}$ \\ ${ }^{1}$ Oxidation Biology Laboratory, Mental Health Research Institute, and 2Department of Pathology, The University of Melbourne, Parkville, Victoria 3010, \\ Australia \\ Review of Hudry et al.
}

Preventing neurotoxicity induced by amyloid- $\beta(\mathrm{A} \beta)$ is central to developing therapies for Alzheimer's disease (AD). However, this peptide has proven difficult to target pharmacologically because it is produced by a complex proteolytic pathway, and the secretases involved in its production have additional substrates that are necessary for normal biological functions. Therefore, intracellular signaling messengers activated by $\mathrm{A} \beta$ may provide more tractable therapeutic targets.

Dysregulation of calcium-mediated signaling pathways have been implicated in $\mathrm{A} \beta$ toxicity. Calcium homeostasis is disrupted in $\mathrm{AD}$ and this is thought to result from interactions between $A \beta$ and NMDA receptors (Snyder et al., 2005). $\mathrm{A} \beta$-induced calcium elevation activates calcineurin $(\mathrm{CaN})$, a phosphatase, which causes dendritic spine atrophy, and inhibition of CaN by AKAP79 rescues the pathology (Wu et al., 2010). However, the

Received May 3, 2012; revised May 28, 2012; accepted June 4, 2012.

This work was supported by funds from the Australian National Health and Medical Research Council and Operational Infrastructure Support from the Victorian state government.

*S.A. and P.L. contributed equally to this work.

Correspondence should be addressed to either Scott Ayton or Peng Lei, Mental Health Research Institute, Kenneth Myer Building, Genetics Lane on Royal Parade, Parkville, Victoria 3010, Australia. E-mail: scott.ayton@unimelb.edu.au or p.lei@mhri.edu.au.

DOI:10.1523/JNEUROSCI.2143-12.2012

Copyright $\odot 2012$ the authors $\quad 0270-6474 / 12 / 329454-03 \$ 15.00 / 0$ mechanism underlying $\mathrm{A} \beta$-induced $\mathrm{CaN}$ activation and how this contributes to neurotoxicity in $\mathrm{AD}$ are unknown.

The nuclear factor of activated T-cells (NFAT) is a downstream messenger induced by CaN (Gómez-Sintes and Lucas, 2010; Wu et al., 2010). NFAT, normally localized in the cytosol, contains a cryptic nuclear localization signal that is revealed upon dephosphorylation by CaN. CaN and NFAT enter the nucleus as a complex where $\mathrm{CaN}$ maintains NFAT in its dephosphorylated state (Zhu and McKeon, 1999). Dephosphorylation masks a nuclear export signal on NFAT to keep the complex within the nucleus (Zhu and McKeon, 1999). Nuclear retention of NFAT promotes transcription of proteins that regulate development, immune function, and, relevant to neurodegeneration, apoptosis (Gómez-Sintes and Lucas, 2010). In AD brains, increased localization of NFAT to the nuclear compartment is associated with elevation of soluble $A \beta$ and cognitive impairment (Abdul et al., 2009).

In a recent issue of The Journal of Neuroscience, Hudry et al. (2012) extend their earlier findings (Wu et al., 2010) to elucidate the $\mathrm{Ca}^{2+}$-CaN-NFAT pathway in models of $\mathrm{AD}$. They recapitulated $\mathrm{AD}$-phenotypical cell morphology (spine loss and dendritic branch simplification) in primary cortical neurons by overexpressing constitutively active forms of either CaN or NFAT. The pathologies evident in both genotypes could be prevented by coexpressing a peptide inhibitor of CaN-NFAT binding, VIVIT, which demonstrated that NFAT is the downstream messenger required for $\mathrm{CaN}$ induced cytotoxicity.

In Hudry et al. (2012), NFAT immunostaining revealed that VIVIT coexpression prevented NFAT from entering the nuclear compartment both in primary cortical neuron and in neurons of mice injected with a vector containing the VIVIT construct. The inhibition of CaNNFAT binding specifically within the nucleus was shown to be sufficient to ameliorate toxicity, as microscopy data revealed that VIVIT engineered with a nuclear localization signal (NLS-VIVIT) prevented both accumulation of NFAT within the nucleus and cytotoxic signaling. Within the nucleus, dephosphorylation by $\mathrm{CaN}$ prevents nuclear export of NFAT (Zhu and McKeon, 1999), which Hudry et al. (2012) argue is inhibited by NLS-VIVIT. As a control, VIVIT with a myristoylation tag, which directed the peptide toward the cell membrane (without the ability to act in the nucleus), was not effective in preventing nuclear NFAT accumulation. Collectively, these data indicate that NFAT induces cell morphological changes by its actions in the nucleus.

Hudry et al. (2012) implicate the CaNNFAT apoptotic pathway in $\mathrm{AD}$ pathogenesis by showing that $\mathrm{A} \beta$ activates 
NFAT and causes toxicity similar to that produced by CaN or NFAT overexpression. The authors harvested primary cortical neurons engineered to overproduce $\mathrm{A} \beta$ from mice expressing $\mathrm{AD}$-linked mutants of the amyloid precursor protein (APP) and presenilin, one of the secretases that cleaves APP to form A $\beta$ (APP/PS1 mice). These neurons exhibited dendritic spine atrophy, which was rescued by VIVIT coexpression. The authors then demonstrated that spine atrophy was specifically caused by $\mathrm{A} \beta$ (as opposed to primarily by presenilin or APP) by showing that treatment with an $\mathrm{A} \beta$ antibody (6E10) attenuated the pathology observed in wild-type cells incubated in media harvested from the transgenic primary neurons.

The CaN-NFAT pathway was also interrogated in vivo in APP/PS1 mice. An adeno-associated virus-based vector was used to transfect VIVIT-GFP, NLSVIVIT-GFP, and GFP (control) constructs into plaque-containing cortex of 7-month-old APP/PS1 and littermate mice by stereotactically injection. A cranial window was implanted in the living animals 1 month later and neurites and spines were quantified by multiphoton imaging. Diminished spine density was observed in the APP/PS1 mice, but was prevented by coexpression of either VIVIT or NLS-VIVIT.

Hudry et al. (2012) conclude, therefore, that $\mathrm{A} \beta$ activates $\mathrm{CaN}$, which in turn promotes nuclear translocation of NFAT to cause cytotoxicity. A previous report described an alternative sequence for this pathway (Cho et al., 2008): NFAT activation increases $A \beta$ production by increasing expression of $\beta$-site APP-cleaving enzyme 1 (BACE1), which proteolitcally cleaves APP to produce the $\mathrm{A} \beta$ peptide. Therefore, the activation of the NFAT pathway might have induced AD-like morphology by increasing endogenous $A \beta$ expression in addition to the pathway suggested by Hudry et al. (2012). These two intersecting pathways presented by Cho et al. (2008) and Hudry et al. (2012) are likely to form a toxic cycle in the pathogenesis of $\mathrm{AD}$ (Fig. 1).

Hudry et al. (2012) used in vitro and in vivo models to describe the nuclear translocation of NFAT as a downstream factor in $\mathrm{A} \beta$-induced cytotoxiciy, which highlights NFAT as a target for $\mathrm{AD}$ pharmacological intervention. In addition, this study has implications for another class of putative $\mathrm{AD}$ therapeutics that target glycogen synthase

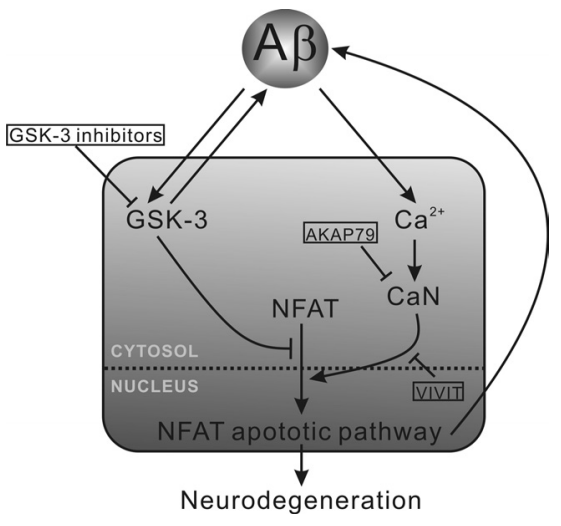

Figure 1. $A \beta$-induced NFAT apoptotic pathway is also activated by GSK-3 inhibition. Calcium elevation induced by $A \beta$ toxicity activates $\mathrm{CaN}$, which causes NFAT trafficking toward, and retention in, the nucleus. NFAT in the nuclear compartment promotes $A \beta$ production (by increasing expression of BACE1) and also signals for apoptosis by increasing expression of Fas (Gómez-Sintes and Lucas, 2010). This apoptotic pathway can be prevented by inhibition of CaN activity (AKAP79) or inhibition of CaNNFAT binding (VIVIT). A $\beta$ also activates GSK-3 and activated GSK-3 promotes $A \beta$ production. Activated GSK-3 prevents NFAT translocation and, consequently, inhibitors of GSK-3 promote NFAT-mediated apoptosis leading to neurodegeneration.

kinase-3 (GSK-3), which should be revisited in light of the data published by Hudry et al. (2012).

GSK-3 is a serine/threonine protein kinase involved in several cellular processes, including the division, proliferation, differentiation, and adhesion of cells (Lei et al., 2011). A $\beta$ activates GSK-3, and inhibition of this kinase reduces $A \beta$ production as well as tau phosphorylation in AD models (Lei et al., 2011). GSK-3 inhibitors, therefore, have been investigated as AD therapeutics, but have shown conflicting outcomes in preclinical and clinical trials (Hampel et al., 2009; Hu et al., 2009; Lei et al., 2011). Pharmacological inhibition of GSK-3 showed some benefit in an intracerebroventricular $\mathrm{A} \beta$ infusion model; however, mice that were administered the drug alone exhibited markers of neurodegeneration (Hu et al., 2009). In addition, the GSK-3 inhibitor lithium was tested in a randomized, placebo-controlled, phase II clinical trial and showed no benefit to cognition for patients with AD (Hampel et al., 2009). Possibly explaining these opposing outcomes, genetic and pharmacological reduction of GSK-3 activity have also been shown to activate NFAT (Ohteki et al., 2000; Gómez-Sintes and Lucas, 2010) and have been associated with NFAT-induced neuronal apoptosis, resulting in brain-wide neurodegen- eration (Gómez-Sintes and Lucas, 2010). If, as shown by Hudry et al. (2012), AD pathogenesis involves NFAT activation by $\mathrm{A} \beta$, then further NFAT activation by pharmacological inhibition of GSK-3 could exaggerate apoptotic signaling in $\mathrm{AD}$ (Fig. 1). The pathway presented by Hudry et al. (2012) might help explain some of the complexity surrounding the results of GSK-3 inhibitors in preclinical and clinical trials.

In summary, the recent paper by Hudry et al. (2012) proposes a pathway of $\mathrm{A} \beta$ toxicity that is mediated by $\mathrm{CaN}$ NFAT apoptotic pathway. Specifically, they show that pathological translocation of NFAT to the nucleus, which is similarly observed in AD brains (Abdul et al., 2009), can be induced by $\mathrm{A} \beta$; therefore, this pathway may be malleable for $\mathrm{AD}$ therapeutic intervention. The inhibition of GSK-3, also an AD-therapeutic strategy, additionally activates the NFAT apoptotic pathway (Ohteki et al., 2000; Gómez-Sintes and Lucas, 2010), leading to neurodegeneration ( $\mathrm{Hu}$ et al., 2009; Gómez-Sintes and Lucas, 2010). The pathway presented by Hudry et al. (2012) illuminates a possible cellular mechanism to explain why cognitive decline was not prevented by GSK-3 inhibition in an AD clinical trial (Hampel et al., 2009). Future research investigating GSK-3 inhibitors should consider the possible side effects of NFAT activation.

\section{References}

Abdul HM, Sama MA, Furman JL, Mathis DM, Beckett TL, Weidner AM, Patel ES, Baig I, Murphy MP, LeVine H 3rd, Kraner SD, Norris CM (2009) Cognitive decline in Alzheimer's disease is associated with selective changes in calcineurin/NFAT signaling. J Neurosci 29:12957-12969.

Cho HJ, Jin SM, Youn HD, Huh K, Mook-Jung I (2008) Disrupted intracellular calcium regulates BACE1 gene expression via nuclear factor of activated T cells 1 (NFAT 1) signaling. Aging Cell 7:137-147.

Gómez-Sintes R, Lucas JJ (2010) NFAT/Fas signaling mediates the neuronal apoptosis and motor side effects of GSK-3 inhibition in a mouse model of lithium therapy. J Clin Invest 120:2432-2445.

Hampel H, Ewers M, Bürger K, Annas P, Mörtberg A, Bogstedt A, Frölich L, Schröder J, Schönknecht P, Riepe MW, Kraft I, Gasser T, Leyhe T, Möller HJ, Kurz A, Basun H (2009) Lithium trial in Alzheimer's disease: a randomized, single-blind, placebo-controlled, multicenter 10-week study. J Clin Psychiatry 70:922-931.

Hu S, Begum AN, Jones MR, Oh MS, Beech WK, Beech BH, Yang F, Chen P, Ubeda OJ, Kim 
PC, Davies P, Ma Q, Cole GM, Frautschy SA (2009) GSK3 inhibitors show benefits in an Alzheimer's disease (AD) model of neurodegeneration but adverse effects in control animals. Neurobiol Dis 33:193-206.

Hudry E, Wu HY, Arbel-Ornath M, Hashimoto T, Matsouaka R, Fan Z, Spires-Jones TL, Betensky RA, Bacskai BJ, Hyman BT (2012) Inhibition of the NFAT pathway alleviates amyloid beta neurotoxicity in a mouse model of Alzheimer's disease. J Neurosci 32:3176-3192.

Lei P, Ayton S, Bush AI, Adlard PA (2011)
GSK-3 in neurodegenerative diseases. Int J Alzheimers Dis 2011:189246.

Ohteki T, Parsons M, Zakarian A, Jones RG, Nguyen LT, Woodgett JR, Ohashi PS (2000) Negative regulation of $\mathrm{T}$ cell proliferation and interleukin 2 production by the serine threonine kinase GSK-3. J Exp Med 192: 99-104.

Snyder EM, Nong Y, Almeida CG, Paul S, Moran T, Choi EY, Nairn AC, Salter MW, Lombroso PJ, Gouras GK, Greengard P (2005) Regulation of NMDA receptor trafficking by amyloid-beta. Nat Neurosci 8:1051-1058.
Wu HY, Hudry E, Hashimoto T, Kuchibhotla K, Rozkalne A, Fan Z, Spires-Jones T, Xie $\mathrm{H}$, Arbel-Ornath M, Grosskreutz CL, Bacskai BJ, Hyman BT (2010) Amyloid beta induces the morphological neurodegenerative triad of spine loss, dendritic simplification, and neuritic dystrophies through calcineurin activation. J Neurosci 30:2636-2649.

Zhu J, McKeon F (1999) NF-AT activation requires suppression of $\mathrm{Crm} 1$-dependent export by calcineurin. Nature 398:256260. 